\title{
Comparison of laparoscopic surgical skills acquired on a virtual reality simulator and a box trainer: an analysis
} for obstetrics-gynecology residents

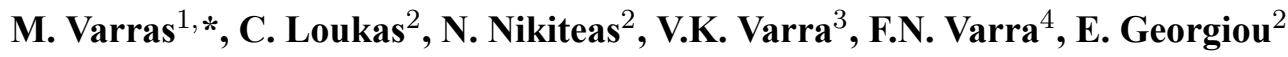 \\ ${ }^{1}$ Fifth Department of Obstetrics and Gynecology, 'Elena Venizelou' General Maternity Hospital, Athens (Greece) \\ ${ }^{2}$ Simulation Center, Medical Physics Laboratory, School of Medicine, National and Kapodistrian University of Athens (Greece) \\ ${ }^{3}$ Department of Pharmacy, University of Patras (Greece) \\ ${ }^{4}$ Department of Pharmacy, Frederick University, Nicosia (Cyprus)
}

\begin{abstract}
Summary
Background/Aims: It is well known that laparoscopic surgery requires the demonstration of a different set of technical skills when compared to open surgery. Laparoscopic training using simulators has been shown to accelerate learning in an efficient and standardized manner. Significant research has been conducted for skills acquisition in abdominal surgery, but in the field of gynecologic laparoscopy the relevant studies are limited. The aim of this study was to compare the training efficacy of virtual reality (VR) simulators and boxtrainers (BTs) for skills acquisition in gynecologic surgery, and also to study the transferability of these skills in the performance of more advanced gynecologic operations. Methods: Twenty residents in obstetrics-gynecology with minimal laparoscopic experience were randomized into two equal groups to be trained on either a VR simulator (Group-A) or a BT (Group-B). Group-A was trained on basic tasks (clipping, peg transfer, and cutting), whereas Group-B was trained on ovarian cystectomy and salpingotomy using custom training models. After training, the two groups were assessed on the performance of two laparoscopic gynecologic procedures on a VR simulator (salpingotomy and salpingectomy). Performance metrics included time, instrument pathlength, and various task-specific errors. Results: Both groups demonstrated significant performance improvement in all training tasks, for all but one of the metrics $(p$ $<0.05)$. After training, both groups had improved performance in the laparoscopic operations using the VR simulator, but this trend was not statistically significant in any metric considered $(p>0.05)$. Similarly, the post-training performance between the two groups was not statistically different $(p>0.05)$. Conclusions: Basic skills training on either a VR simulator or BT results in equivalent but not statistically significant performance improvement with more advanced gynecologic laparoscopic tasks on a VR simulator.
\end{abstract}

Key words: Virtual reality simulator; Box-trainer; Gynecologic surgical training; Ectopic pregnancy; Salpingotomy; Salpingectomy.

\section{Introduction}

Laparoscopic surgery (LS) is the standard technique for an increasing number of operations. Some of the major benefits compared to open surgery include: minimized risk of infection, reduced postoperative pain, shorter rehabilitation time, and better cosmetic results. In practice, LS requires a very different set of both technical and non-technical skills when compared to open surgery, designed to address the absence of $3 \mathrm{D}$ vision, the use of long and thin instruments, the fulcrum effect, and limited force feedback [1-3].

Realistic anatomical models and human cadavers have been employed by surgeons to practice the various psychomotor and visuospatial skills required to perform LS procedures. However, these models need frequent replacement and have high maintenance costs [4]. Over the last decade, simulation-based surgical education has received significant attention, and a number of laparoscopic simulators have been employed [5]. Box trainers (BTs) and virtual reality (VR) simulators are the two main training modalities employed in the hospitals and clinical training centers for laparoscopic skills acquisition and development. A number of studies have demonstrated that training with such devices improves the required laparoscopic skills and that these skills are transferable to the operating room [6-8]. High-fidelity models with life-like patient anatomy are employed for the development of special psychomotor skills outside the operating theater [9]. Trainees are able to develop surgical skills and become familiar with a particular procedure in a surgical laboratory before operating on a patient [10-11]. However, there remain some questions about the superiority of laparoscopic VR simulators over BTs with regard to the level of training and the skills achieved, mainly due to the different sense of force-feedback, the difference in the image quality, the behavior of the tissue models, as well as the dissimilarity of the tasks performed with the two modalities [12-13].

A significant amount of research has been published evaluating simulation-based training for general surgery procedures and gynecology. Gor et al. suggested that the MIST-VR simulator provides objective assessment of the
Clin. Exp. Obstet. Gynecol. - ISSN: 0390-6663 XLVII, n. 5, 2020

doi: $10.31083 /$ j.ceog.2020.05.4988
This is an open access article under the CC BY 4.0 license (https://creativecommons.org/licenses/by/4.0/). 

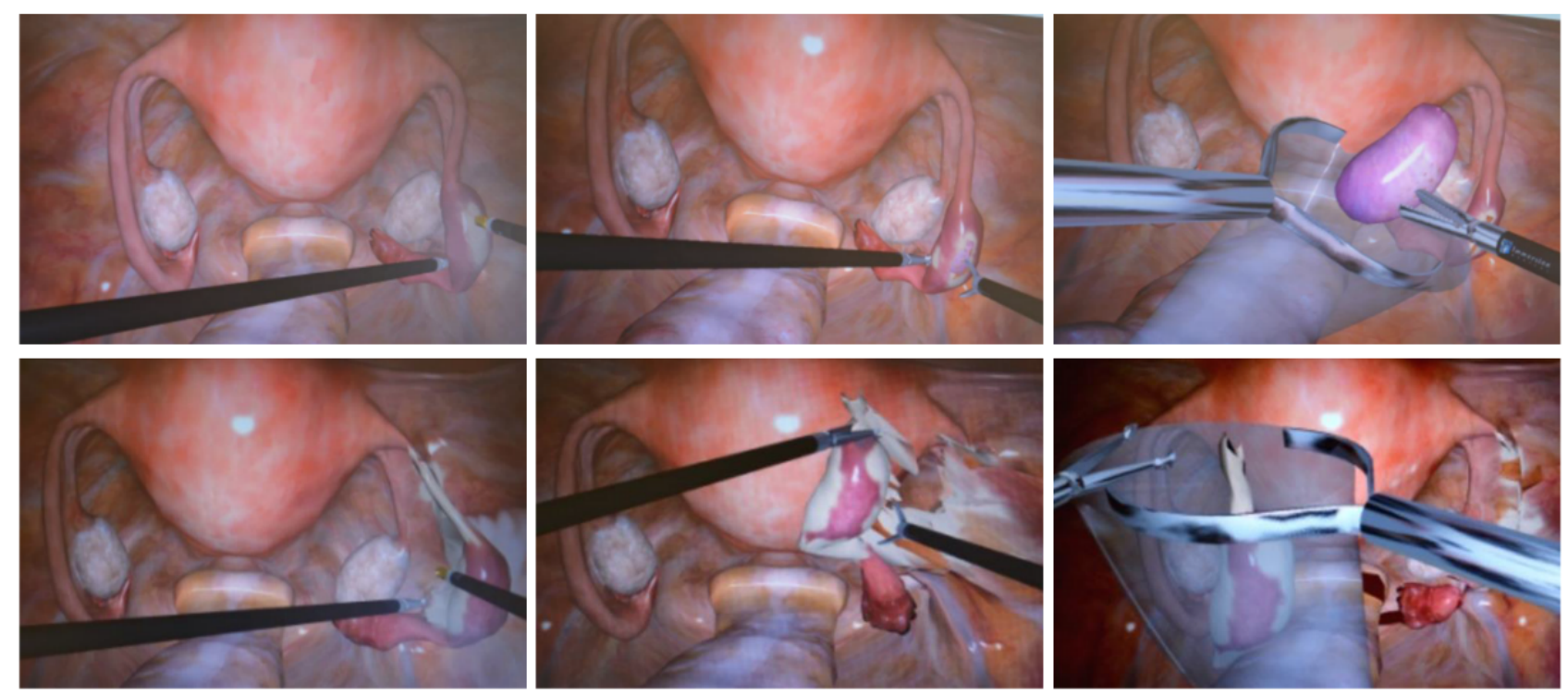

Figure 1. - Various steps from the salpingotomy (top) and salpingectomy (bottom) procedures performed on the VR simulator.

laparoscopic skills of gynecologists [14]. Aggarwal et al. demonstrated that an ectopic pregnancy scenario in a VR simulator can differentiate $\mathrm{Ob} / \mathrm{Gyn}$ residents with different level of surgical experience [15]. Similarly, Larsen et al. showed that expert gynecologists outperformed intermediates and novices in time, instrument pathlength, and total errors [16]. Schreuder et al. reported that gynecologists with minimal laparoscopic experience can improve their skills during short-phase training on a VR procedural simulator [17].

Investigations of the training value of BTs is more focused on the development of appropriate anatomic models for gynecologic surgeries. For example, Scott et al. investigated the laparoscopic cost effectiveness of bench models in relation to operating room experience [18]. Zhen et al. highlighted the use of the fundamentals of laparoscopic surgery (FLS) program to assess the laparoscopic competence of gynecologists [19]. Tang et al. described the design of a phantom that allows trainees to practice key skills required for laparoscopic salpingotomy and salpingectomy [20]. A porcine small bowel was used to simulate the fallopian tube, while porcine liver and red food dye were used to simulate ectopic pregnancies inside the fallopian tube. Levine et al. described the use of a lightly embalmed human cadaver model for practicing laparoscopic techniques for various operations, including laparoscopic hysterectomy [21]. A live porcine model for teaching advanced laparoscopic skills to gynecologic oncology fellows was developed by Hoffman et al. [22]. The model was found to be suitable for laparoscopic training in various procedures such as lymphadenectomy, uretero-neocystostomy, and bowel anastomoses.

The aim of our study was to compare the training efficacy of two LS simulators and also to assess the transferability of skills acquired, during training on basic laparo- scopic tasks, to the performance of more advanced gynecologic operations on a VR simulator. To achieve this goal we used two separate groups of residents and studied the impact of basic skills training using a VR simulator or a box trainer, using custom training models that represented female gynecological anatomy. Following this training, the two groups were assessed on the performance of two laparoscopic gynecologic operations on a VR simulator (salpingotomy and salpingectomy). The post-training performance was compared to that before training and also compared between the two groups. Our analysis also included investigation of the effect of gender, video gaming skills and musical instrument ability in the performance of laparoscopic operations.

\section{Materials and Methods}

\section{Study design}

Twenty residents in Obstetrics-Gynecology from the 'Elena Venizelou' General Maternity State Hospital were recruited for voluntary participation in the study, which was carried out in the Medical Physics Lab-Simulation Center (MPLSC) of the University of Athens, Greece. All subjects had minimal experience in laparoscopic surgery $(<10$ operations), and no experience with laparoscopic simulators. Written informed consent was obtained prior to participation and our institutional review board granted exception for this study. The participants were randomized into two equal groups to undertake training on either a VR simulator (Group-A) or a BT (Group-B). Subjects within one group were not allowed to practice on the opposing trainers.

After familiarization with the equipment, the participants first performed two laparoscopic operations (salpingotomy and salpingectomy for ectopic pregnancy) on the VR simulator as a pre-training assessment. Group-A was then trained in three VR basic tasks (clipping, peg transfer 

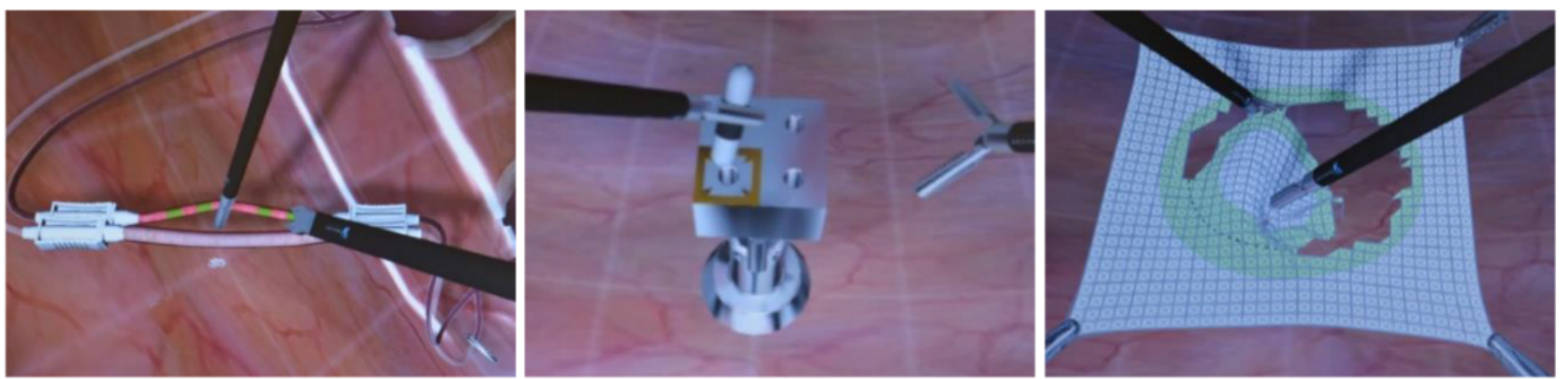

Figure 2. - The three VR training tasks performed by Group A.

and cutting), whereas Group-B practiced two tasks based on custom training models ('cystectomy' for an ovarian cyst, and 'salpingotomy' for an ectopic pregnancy). More details about the training and assessment tasks are provided below. After training sessions, lasting two hours each on two consecutive days, all subjects were re-assessed on the initial VR operations (post-training assessment). Care was taken so that participants from each group received the same duration of training across the tasks.

\section{Assessment and training tasks \\ Ectopic pregnancy on the VR simulator}

The ectopic pregnancy procedures (pre- and postassessment tasks in the study) are based on high-fidelity virtual graphics of the internal female genitalia as would be seen from a camera inserted through the umbilicus. The ectopic pregnancy is placed in the right fallopian tube.

In the 'salpingotomy' scenario (Figure 1), the user needs to grasp and hold the tube on its anti-mesosalpingeal border either proximal or distal to the ectopic section. Then the dominant hand instrument is changed to a cutting device for making the incision. A $2 \mathrm{~cm}$ longitudinal incision is made on the anti-mesosalpingeal border over the proximal portion of the ectopic pregnancy site. If the pregnancy does not protrude after making the incision, the user may make an instrument change to the suction-irrigation device and attempt hydro-dissection with saline. Finally, the user places the tissue removed in a retrieval bag. Performance metrics were: time for cautery used, total blood loss, incision length, total instrument pathlength, and time.

In the 'salpingectomy scenario' (Figure 1), an ectopic pregnancy with tubal adhesions has to be dissected from the fallopian tube, the adhesions and the surrounding membranes. The user begins just distal to the cornual area of the affected tube using bipolar electrosurgery and coagulates 2-3 successive overlapping passes until a 2- to 3-cm length is desiccated. Then the infundibulo-ovarian ligament is identified and coagulated. Using scissors, the desiccated region is cut in the middle. Beginning at either end, the user starts the division of the mesosalpinx using bipolar electrosurgery, staying close to the fallopian tube, and finally places the tube with the excised ectopic in specimen bag. Performance metrics include: time used for cautery, total blood loss, adhesions ripped (due to excessive force), adhesions lysed, instrument pathlength, and time.

\section{Training tasks on the VR simulator (Group-A)}

The selected training tasks are common in laparoscopic simulation training, presenting a gradual increase in complexity and a variability of psychomotor skills (see Figure 2). The first task, clipping, required the user to apply 4 clips at pre-marked positions on each end of the vessel. Once the clips are adequately placed, a mark appears on the vessel between the clips indicating the area to cut. Performance was measured as: number of clips applied, number of clips dropped, instrument pathlength, and time.

The second training task (peg transfer), required that the user pick up a series of four cylindrical pegs from the floor of the cavity and place them into the correct holes of a pegboard. For two of the four pegs, the task required peg transfer between the graspers. Performance metrics included: number of pegs dropped, instrument pathlength, and time.

Cutting was the third training task and required the user to cut a section of gauze along the perimeter of a circle and within a boundary area that indicated the maximum allowable deviation. Excessive tension on the gauze leads to avulsion from the anchoring hooks. The simulation ended when the circular shape was completely cut out and the excised cloth was completely freed from the original piece. Performance was measured as: percentage cutting out of boundary area, number of unsuccessful cutting attempts, instrument pathlength, and time.

\section{Training tasks on the BT (Group-B)}

The first task was a simulated ovarian cystectomy as shown in Figure 3. The training model was a medium-sized balloon filled with clay and tied at its end. This balloon was put inside another balloon to simulate the ovarian cortex. A 7-cm vertical black line was marked on the outer balloon. The model was fixed inside the box trainer. The model served as a replica of cystectomy, such as in ovariancyst surgery. The task required the trainee to cut the outer balloon on the marked line avoiding cutting of the inner balloon, which represents the ovarian cyst. Minimal damage of the inner balloon was taken into consideration. Per- 

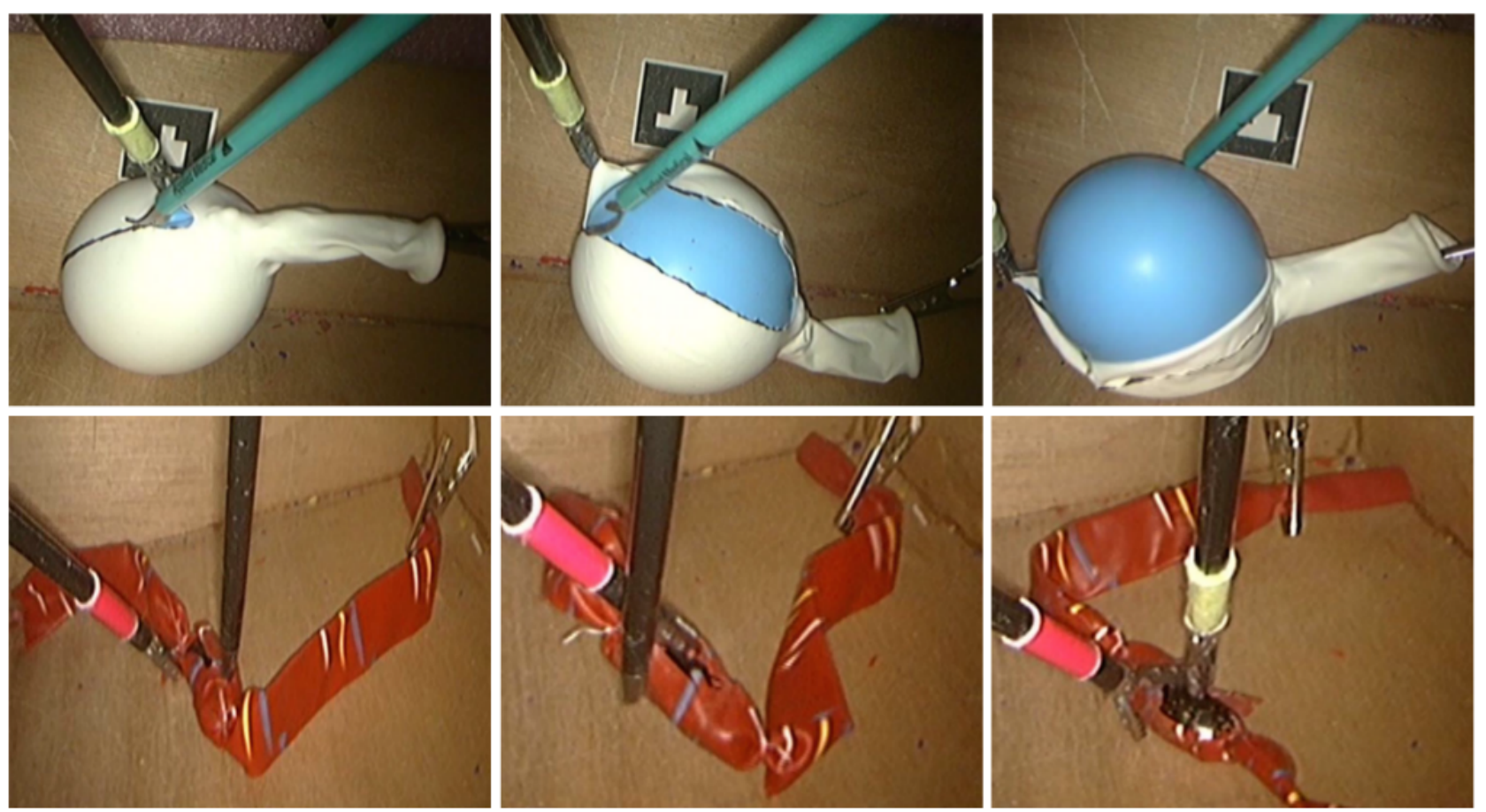

Figure 3. - Various steps from the two training tasks (top: cystectomy, bottom: salpingotomy), performed by Group B on the BT.

formance metrics included: minimal damage of the "cystic wall' (yes/no), a successful 7-cm longitudinal incision on the 'ovarian cortex' (yes/no), maximum deviation from the labeled line, instrument pathlength, and time.

The second task was a simulated salpingotomy as pictured in Figure 3. The fallopian tube ectopic pregnancy model was composed of a $15-\mathrm{cm}$ oblong balloon with a 2$\mathrm{cm}$ long bean put inside to simulate the trophoblastic tissue. The balloon was sewn at both ends of the bean using a thin thread. The model was fixed inside the box trainer. The model served as a replica of salpingotomy for extraction of the trophoblastic tissue as performed in laparoscopic conservative ectopic surgery. The trainee had to make a longitudinal incision on the balloon and extract the bean. Performance was measured as: success with the longitudinal incision (yes/no), total instrument pathlength, and time. At this point it should be noted that both tasks were captured on video and performances were scored by an expert investigator in a blinded fashion. Instrument pathlength was computed via custom video analysis software, as reported in another study [23].

\section{Equipment}

The VR simulator was the Immersion laparoscopic LapVR simulator (Immersion Inc., San Jose, CA, USA). LapVR has been validated as an effective educational laparoscopic tool in prior studies [23-25]. The laparoscopic BT has been described previously [23]. In brief, the BT simulator includes a pelvic trainer, a rigid laparoscope with zero degrees angle of view and an optical fiber light ca- ble connected to an Olympus (Hamburg, Germany) laparoscopic tower (19-in. flat screen, 3CCD digital processor and xenon light source, and DVD recorder).

\section{Statistics}

Statistical analysis was performed using SPSS 20.0 software for Windows (SPSS Inc., Chicago, IL, USA). Categorical variables were compared by chi-squared test. Significance of differences in the measurements between/within groups was determined by Mann-Whitney U test. The correlation between performance scores and number of repetitions across each task was statistically assessed using the nonparametric Spearman's correlation analysis. A $p$-value of 0.05 or less was considered statistically significant.

\section{Results}

\section{Subject demographics}

Group-A included 6 men $(60 \%)$ and 4 women $(40 \%)$ with a median age of 34 years. In this group 4 residents $(40 \%)$ identified themselves as video gamers and $3(30 \%)$ as playing musical instruments. Group B included 5 men $(50 \%)$ and 5 women $(50 \%)$ with a mean age of 32 years. In Group-B 4 residents $(40 \%)$ had experience with video gaming and $3(30 \%)$ played musical instruments.

\section{Training performance}

Subjects who trained on the VR simulator (Group-A) demonstrated performance improvement for almost all metrics assessed in each task (Tables 1-3). Specifically, on the last two repetitions the performances were improved when 
Table 1. - Group A, 'Clipping': Training results (mean \pm standard deviation), and correlation coefficient w.r.t. to the number of repetitions performed (VR simulator). Bold numbers indicate significance.

\begin{tabular}{lccccc}
\hline & $\begin{array}{c}\text { First two } \\
\text { repetitions }\end{array}$ & $\begin{array}{c}\text { Last two } \\
\text { repetitions }\end{array}$ & $p$-values & Correlation coefficient & $p$-values \\
\hline Number of clips applied & $4.60 \pm 2.68$ & $4.10 \pm 0.45$ & 0.42 & -0.02 & 0.77 \\
Number of clips dropped & $0.85 \pm 0.23$ & $0.07 \pm 0.26$ & $\mathbf{0 . 0 1}$ & -0.18 & $<\mathbf{0 . 0 1}$ \\
Total instrument pathlength (m) & $2.45 \pm 1.50$ & $1.62 \pm 0.64$ & $\mathbf{0 . 0 4}$ & -0.36 & $<\mathbf{0 . 0 1}$ \\
Completion time (s) & $149 \pm 65$ & $89 \pm 40$ & $<\mathbf{0 . 0 1 0}$ & -0.46 & $<\mathbf{0 . 0 1}$ \\
\hline
\end{tabular}

Table 2. - Group A, 'Peg transfer': Training results (mean \pm standard deviation), and correlation coefficient w.r.t. to the number of repetitions performed (VR simulator). Bold numbers indicate significance.

\begin{tabular}{lccccc}
\hline & First two repetitions Last two repetitions $p$-values & Correlation coefficient $p$-values \\
\hline Number of pegs dropped & $1.10 \pm 0.85$ & $0.42 \pm 0.63$ & $\mathbf{0 . 0 2}$ & -0.22 & $<\mathbf{0 . 0 1}$ \\
Total instrument pathlength (m) & $6.47 \pm 1.36$ & $4.41 \pm 0.57$ & $<\mathbf{0 . 0 1}$ & -0.27 & $<\mathbf{0 . 0 1}$ \\
Completion time (s) & $184 \pm 80$ & $104 \pm 30$ & $<\mathbf{0 . 0 1}$ & -0.51 & $<\mathbf{0 . 0 1}$ \\
\hline
\end{tabular}

Table 3. - Group A, 'Cutting': Training results (mean \pm standard deviation), and correlation coefficient w.r.t. to the number of repetitions performed (VR simulator). Bold numbers indicate significance.

\begin{tabular}{lcccc}
\hline & \multicolumn{2}{c}{ First two repetitions } & Last two repetitions $p$-values Correlation coefficient $p$-values \\
\hline Percentage cutting out of boundary area & $0.70 \pm 1.38$ & $0.47 \pm 0.67$ & $\mathbf{0 . 0 4}$ & -0.29 \\
Number of unsuccessful cutting attempts & $5.90 \pm 6.67$ & $1.80 \pm 2.71$ & $\mathbf{0 . 0 2}$ & -0.19 \\
Total instrument pathlength (m) & $7.43 \pm 2.12$ & $4.25 \pm 2.15$ & $\mathbf{0 . 0 4}$ & -0.33 \\
Completion time (s) & $294 \pm 147$ & $166 \pm 67$ & $<\mathbf{0 . 0 1}$ & -0.53 \\
\hline
\end{tabular}

compared to the first two attempts. For clipping the performance was improved for 3 out of the 4 metrics: number of clips dropped, instrument pathlength, and time. For the other tasks the performance was improved in all metrics assessed (peg transfer: number of pegs dropped, instrument pathlength and time; cutting: percentage cutting out of boundary area, number of unsuccessful cutting attempts, instrument pathlength and time). It is worth noting also that 'completion time' increased across the three tasks, denoting their increased difficulty in progressing from clipping to peg transfer to cutting. Moreover, a statistically significant negative correlation was found between the metrics and the number of repetitions performed in almost all metrics for each task (clipping: in 3 out of 4 metrics, peg transfer: in all 3 metrics, and cutting: in all 4 metrics). The correlation was found to be statistically significant for the same metrics, described previously, that showed significant improvement between the first and the last repetitions. The negative sign for the correlation coefficient denotes improvement over the training period.

For Group-B (trained on the BT), the performances were improved on the last two repetitions when compared to the first two attempts. Specifically, a statistically significant performance improvement was found for almost all performance metrics in each task (Tables 4-5). For ovarian cystectomy the significant improvement was found for 4 out of 5 metrics: minimal damage of the cystic wall, success for a 7-cm longitudinal incision on the ovarian cortex, maximum deviation from the labelled line, instrument pathlength, and time. For salpingotomy, success of longitudinal incision, instrument pathlength and time were significantly improved. Moreover, in each task a statistically significant negative correlation was found between the statistically improved metrics and the number of repetitions performed.

\section{Training assessment}

Prior to training the two groups had equivalent performance in the two assessment tasks performed on the VR simulator $(p>0.05$, results not shown here). Tables 6-7 show for each group the comparison of the results before and after training for salpingotomy and salpingectomy respectively. Although the trainees' performance on both VR tasks improved after training, this improvement was not statistically different $(p>0.05)$.

Our analysis also investigated the effect of gender, video gaming and music instrument ability in the performance of laparoscopic operations. For salpingotomy, the only statistically significant difference was found for video game players with respect to the improved length of the incision parameter, during post-training $(p=0.039)$. For salpingectomy, video gamers also showed a statistically significant improvement, again with respect to the instrument pathlength parameter $(p=0.033)$. No other statistically significant difference was found in this investigation. 
Table 4. - Group B, 'Ovarian cystectomy': Training results (mean \pm standard deviation), and correlation coefficient w.r.t. to the number of repetitions performed (Box Trainer). Bold numbers indicate significance.

\begin{tabular}{|c|c|c|c|c|c|}
\hline & First two repetitions & Last two repetitions & $p$-values & Correlation coefficient & $p$-values \\
\hline $\begin{array}{l}\text { Minimal damage of the cys- } \\
\text { tic wall }(\text { yes }=1 / \text { no }=2)\end{array}$ & $1.95 \pm 0.22$ & $1.60 \pm 0.30$ & 0.04 & -0.4 & $<\mathbf{0 . 0 1}$ \\
\hline $\begin{array}{l}\text { Success for a } 7 \text {-cm longitu- } \\
\text { dinal } \\
\text { incision on the ovarian cor- } \\
\text { tex }(y=1 / \mathrm{n}=2)\end{array}$ & $1.45 \pm 0.21$ & $1.10 \pm 0.27$ & 0.04 & -0.18 & 0.04 \\
\hline $\begin{array}{l}\text { Maximum deviation from } \\
\text { the labelled line }(\mathrm{mm})\end{array}$ & $1.57 \pm 0.95$ & $1.50 \pm 0.98$ & 0.58 & -0.14 & 0.10 \\
\hline $\begin{array}{l}\text { Total instrument pathlength } \\
\text { (m) }\end{array}$ & $13.42 \pm 7.22$ & $9.66 \pm 5.05$ & 0.03 & -0.22 & 0.01 \\
\hline Completion time (s) & $480 \pm 110$ & $320 \pm 136$ & 0.02 & -0.19 & 0.04 \\
\hline
\end{tabular}

Table 5. - Group B, 'Salpingotomy': Training results (mean \pm standard deviation), and correlation coefficient w.r.t. to the number of repetitions performed (Box Trainer). Bold numbers indicate significance.

\begin{tabular}{lccccc}
\hline & First two repetitions & Last two repetitions & $p$-values & Correlation coefficient & $p$-values \\
\hline Success of longitudinal & $1.53 \pm 0.51$ & $1.26 \pm 0.45$ & 0.17 & -0.24 & $\mathbf{0 . 0 3}$ \\
incision $(\mathrm{y}=1 / \mathrm{n}=2)$ & $10.98 \pm 5.66$ & $4.30 \pm 2.11$ & $<\mathbf{0 . 0 1}$ & -0.65 & $<\mathbf{0 . 0 1}$ \\
Total instrument pathlength (a.u.) & $382 \pm 183$ & $136 \pm 65$ & $<\mathbf{0 . 0 1}$ & -0.66 & $<\mathbf{0 . 0 1}$ \\
Completion time (s) & & & & & \\
\hline
\end{tabular}

Table 6. - Pre-training vs. post-training performance results (mean \pm standard deviation), for each group,

\begin{tabular}{lccc}
\multicolumn{4}{c}{ for the salpingotomy assessment task performed on the VR simulator. } \\
\hline & Pre-training & Post-training & $p$-values \\
\hline Group A & & & \\
Time for cautery used (s) & $38.51 \pm 30.44$ & $33.61 \pm 32.17$ & 0.91 \\
Total blood loss (cc) & $69.40 \pm 31.06$ & $60.64 \pm 18.24$ & 0.18 \\
Incision length (cm) & $2.42 \pm 0.46$ & $2.26 \pm 0.67$ & 0.35 \\
Total instrument pathlength (m) & $6.60 \pm 2.85$ & $6.57 \pm 4.32$ & 0.43 \\
Completion time (s) & $260 \pm 141$ & $222 \pm 120$ & 0.85 \\
Group B & & & \\
Time for cautery used (s) & $50.24 \pm 33.89$ & $28.89 \pm 19.60$ & 0.74 \\
Total blood loss (cc) & $67.56 \pm 28.12$ & $62.45 \pm 22.34$ & 0.68 \\
Incision length (cm) & $2.68 \pm 0.98$ & $2.33 \pm 0.52$ & 0.63 \\
Total instrument pathlength (m) & $6.29 \pm 2.13$ & $7.64 \pm 4.89$ & 0.85 \\
Completion time (s) & $276 \pm 114$ & $252 \pm 164$ & 0.48
\end{tabular}

\section{Discussion}

The increasing popularity of minimally invasive surgery highlights the necessity to develop training programs for improvement of key psychomotor skills such as hand eye coordination. Clinical experience has shown that these skills require a significant training period in the operating theater (10 to 30 patients, depending on the operation), resulting in longer operating times, higher complication rates, higher conversion rate to open laparotomy, and subsequently higher hospital costs [26-27]. Therefore, training using laparoscopic simulators has significant advantages over training on patients, providing a safe and controlled environment without the risk of harm to patients and avoiding the stressful operating conditions with patients.

In this study we aimed to investigate the differential impact of training and skills acquisition in high-fidelity VR simulator vs. low-fidelity BT. The evaluation was conducted on the VR simulator, since there are controversies about the transferability of skills between different laparoscopic training modalities. It has been suggested that the VR simulators are able to assess the skill level of laparoscopic surgeons [28-29]. It also seems that VR simulators, when used appropriately, more closely simulate real laparoscopic procedures [30]. However, VR simulators and 
Table 7. - Pre-training vs. post-training performance results (mean \pm standard deviation), for each group, for the salpingectomy assessment task performed on the VR simulator.

\begin{tabular}{lccc}
\hline & Pre-training & Post-training & $p$-values \\
\hline Group A & & & \\
Time for cautery used (s) & $157.79 \pm 90.65$ & $119.66 \pm 71.36$ & 0.31 \\
Total blood loss (cc) & $585.27 \pm 341.84$ & $405.87 \pm 210.23$ & 0.17 \\
Adhesions ripped (\%) & $2.84 \pm 3.76$ & $2.60 \pm 1.65$ & 0.85 \\
Adhesions lysed (\%) & $97.40 \pm 7.54$ & $94.30 \pm 18.02$ & 0.62 \\
Total instrument pathlength (m) & $20.37 \pm 8.09$ & $19.75 \pm 9.52$ & 0.43 \\
Completion time (s) & $732 \pm 292$ & $650 \pm 318$ & 0.87 \\
Group B & & & \\
Time for cautery used (s) & $131.81 \pm 56.47$ & $159.55 \pm 88.46$ & 0.41 \\
Total blood loss (cc) & $505.97 \pm 279.15$ & $398.05 \pm 211.47$ & 0.34 \\
Adhesions ripped (\%) & $2.68 \pm 0.98$ & $2.33 \pm 0.52$ & 0.84 \\
Adhesions lysed (\%) & $99.40 \pm 0.97$ & $99.80 \pm 0.63$ & 0.28 \\
Total instrument pathlength (m) & $20.33 \pm 7.27$ & $22.31 \pm 9.72$ & 0.61 \\
Completion time (s) & $810 \pm 306$ & $726 \pm 277$ & 0.53 \\
\hline
\end{tabular}

BTs have some fundamental inadequacies. For example, VR simulators provide worse depth perception compared to BTs, whereas in some tasks the properties of the graphical models are not so realistic. On the other hand, BTs provide no automated means for performance assessment, whereas the training models require replacement after a task performance, and maintenance.

In this study the training tasks were not identical for the two groups, as there is no consensus on which tasks to include in a basic laparoscopic training program in order to achieve the most effective skills training. A previous study has shown that training on the same tasks, but using different modalities (VR simulator and BT), resulted in almost equivalent performance [23]. In the present study the tasks on the VR simulator were common basic laparoscopic exercises, whereas the ones in the BT were chosen as more advanced: 'cystectomy' for ovarian cyst, and 'salpingotomy' for ectopic pregnancy. Our main purpose was to investigate whether practice of more advanced tasks on the BT is more effective than VR basic skills training, given that the assessment tasks are simulated surgical operations.

Our results indicated that VR training improves certain laparoscopic skills, in agreement with other studies which have shown that training on basic tasks has a significant impact in the improvement of more complex tasks [29]. In another study it was shown that VR training contributes to the enhancement of key laparoscopic skills, and that experienced surgeons scored better than residents in terms of time and instrument pathlength [25]. Iwata et al. found that basic laparoscopic tasks such as peg transfer and cutting were strong discriminators of laparoscopic experience [24]. Furthermore, Mansour et al. assessed technical and dexterity skills of surgeons by measuring various parameters during peg transfer and cutting, and they found improvement in some aspects of the laparoscopic surgical skills of the trainees [31].
Also in our study, the performance of the subjects improved significantly as a result of the BT training on the two advanced tasks. These exercises were designed to incorporate laparoscopic grasping and cutting skills, which are fundamental for performing laparoscopic ectopic pregnancy surgery. Although the primary goal of training was to increase technical performance (i.e. shorter time and fewer errors), we also observed a decrease in the dexterity variability, as demonstrated by the greater economy of movements performed (i.e. shorter instrument pathlength). This finding was also valid for the other group trained on the VR simulator.

With regard to the pre- and post-training assessments performed on the VR simulator, there was no statistically significant improvement for either of the groups in any performance metric with the laparoscopic operations. Also there was no statistically significant difference between the two groups for any metric. This result may seem surprising, since one would expect the skills acquired during training would be transferred to the performance of the (more advanced) assessment tasks, and/or that one of the two types of training provided would be superior. There are a number of potential reasons for this discrepancy. First, the assessment tasks are actual laparoscopic operations which require the combinative demonstration of various complex skills. Second, in the training sessions, each group practiced a limited number of basic skills separately (such as grasping, cutting, etc.). Thus, when the subjects attempted to perform the laparoscopic operations on the VR simulator, they could not combine the separate skills learned during training. Third, laparoscopic operations require management of additional skills beyond those practiced during basic skills training.

The identical post-training performance of the two groups suggests that VR simulators and BTs are equivalent modalities for laparoscopic basic skill training. One of the limitations of our study could be the fact that both 
groups were assessed before training and after training in VR simulators and that this might provide a positive bias for residents that trained with VR simulators over those using the BTs. However, we chose VR operations for pre- and post-training assessment as we were not able to assess the groups in real surgical operations. It does appear that the bias for the residents who were trained in VR simulators was small since the skills required to perform VR operations are much more advanced than those required for the VR basic tasks training. In a related study Loukas et al. performed a head-to-head comparison study which showed that both modalities provided significant enhancement in novices' performance and that the skills learned on one device were transferable to the other [23]. Other studies have also attempted to clarify which training modality is the most effective by comparing the transferability of skills between VR simulators and BTs $[2,32]$. However, the reported results are contradictory mainly because of the dissimilarity of the tasks performed on each device.

Finally, with respect to the subjects' video gaming and music expertise, only video gaming seemed to have a positive effect in the performance of the laparoscopic operations on the VR simulator. Grantcharov et al. suggests that trainees who regularly play computer games make fewer errors and have shorter learning times [33]. Also, it has been suggested that video gamers acquire laparoscopic techniques faster, and training on video games appears to improve laparoscopic performance with VR trainers [34].

In a review article, we provided an overview of the evaluation and capacity of the different types of laparoscopic simulators including laparoscopic box trainers, laparoscopic VR simulators, animal models, human cadavers and lightly embalmed human cadavers in the development and assessment of training in laparoscopic gynecologic procedures. We suggested that the clinical training curriculum of obstetrician-gynecologists should include laparoscopic VR simulators through a simulation-based education program outside the live operative environment under appropriated supervision in order to ensure the acquisition of maximal surgical psychomotor skills and the achievement of high-quality laparoscopic training [35]. Moreover, Papanikolaou et al. suggested that teaching hospitals should introduce training programs using laparoscopic simulators with standardized and reproducible tasks in order to achieve better patient care with safety, efficiency and lower cost [36]. Furthermore, Torricelli et al. suggested that the best way for the dissemination of laparoscopic surgery in obstetrics-gynecology residents is the induction of laparoscopic simulators for a short period of training [37].

\section{Conclusions}

The present randomized-prospective study showed that basic skills are improved by training with either VR simulators or BTs. However, groups trained with these devices show no statistically significant improvement in laparoscopic operations in a VR simulator. We propose la- paroscopic training laboratories to include VR simulators as a reasonable alternative to BTs for laparoscopic training of inexperienced residents. Compared to BTs, VR simulators provide various means for automated assessment of training performance via a rich set of assessment metrics. These parameters may then be used either by the trainees to evaluate their training progress or by the tutor to adapt the training procedure according to the needs of each individual trainee.

\section{Acknowledgments}

This study was a part of the Thesis for a Master Degree (M.Sc.) in "Minimally Invasive Surgery, Robotic Surgery and Telesurgery”, Medical School, National and Kapodistrian University of Athens from Mr. Michail Varras with tutors the Professor Mr. Nikolaos Nikiteas, and the Assistant Professor Dr. Constantinos Loukas, Medical School, National and Kapodistrian University of Athens.

\section{Conflict of Interest}

The authors declare no conflict of interest.

Submitted: September 23, 2018

Accepted: February 04, 2019

Published: October 15, 2020

\section{References}

[1] Halvorsen F.H., Elle O.J., Fosse E.: "Simulators in surgery". Minim Invasive Ther Allied Technol., 2005, 14, 214-223.

[2] Madan A.K., Frantzides C.T..: "Prospective, randomized controlled trial of laparoscopic trainers in laparoscopic skills acquisition". Surg Endosc, 2007, 21, 209-213.

[3] Hasson H.M., Kumari N.V., Eekhout J.: "Training simulator for developing laparoscopic skills". JSLS, 2001, 5, 255-265.

[4] Bridges M., Diamond D.L.: "The financial impact of teaching surgical residents in the operating room". Am J Surg, 1999, 177, 28-32.

[5] Yiannakopoulou E., Nikiteas N., Perrea D., Tsigris C.: "Virtual reality simulators and training in laparoscopic surgery". Int J Surg, 2015, 13, 60-64.

[6] Madan A.K., Harper J.L., Taddeucci R.J., Tichansky D.S.: "Goaldirected laparoscopic training leads to better laparoscopic skill acquisition". Surgery, 2008, 144, 345-350.

[7] Condous G., Alhamdan D., Bignardi T., Van Calster B., Van Huffel S., Timmerman D., et al.: "The value of laparoscopic skills courses". Aust NZ J Obstet Gynecol, 2009, 49, 312-315.

[8] Hiemstra E., Kolkman W., van de Put M.A.J., Jansen F.W.: "Retention of basic laparoscopic skills after a structured training program". Gynecol Surg, 2009, 6, 229-235.

[9] Rosser J.C., Murayama M., Gabriel N.H.: "Minimally invasive surgical training solutions for the twenty-first century". Surg Clin North Am, 2000, 80, 1607-1624.

[10] Torkington J., Smith S.G., Rees B., Darzi A.: "Skill transfer from virtual reality to a real laparoscopic task". Surg Endosc, 2001, 15, 1076-1079.

[11] Torkington J., Smith S.G., Rees B., Darzi A.: "The role of the basic surgical skills course in the acquisition and retention of laparoscopic skill". Surg Endosc, 2001, 15, 1071-1075.

[12] Hamilton E.C., Scott D.J., Fleming J.B., Rege R.V., Laycock R., Bergen P.C., et al.: "Comparison of video trainer and virtual reality training systems on acquisition of laparoscopic skills. Surg Endosc, 2002, 16, 406-411.

[13] Munz Y., Kumar B.D., Moorthy K., Bann S., Darzi A.: "Laparoscopic virtual reality and box trainers is one superior to the other?". Surg Endosc, 2004, 18, 485-494.

[14] Gor M., McCloy R., Stone R., Smith A.: "Virtual reality laparo- 
scopic simulator for assessment in gynaecology". BJOG, 2003, 110, 181-187.

[15] Aggarwal R., Tully A., Grantcharov T., Larsen C.R., Miskry T., Farthing A., et al.: "Virtual reality simulation training can improve technical skills during laparoscopic salpingectomy for ectopic pregnancy". BJOG, 2006, 113, 1382-1387.

[16] Larsen C.R., Grantcharov T., Aggarwal R., Tully A., Sørensen J.L., Dalsgaard T., et al:: "Objective assessment of gynecologic laparoscopic skills using the LapSimGyn virtual reality simulator". Surg Endosc, 2006, 20, 1460-1466.

[17] Schreuder H.W.R., van Hove P.D., Janse J.A., Verheijen R.R., Stassen L.P., Dankelman J.: “An 'intermediate curriculum' for advanced laparoscopic skills training with virtual reality simulation". J Minim Invasive Gynecol, 2011, 18, 597-606.

[18] Scott D.J., Bergen P.C., Rege R.V., Laycock R., Tesfay S.T., Valentine R.J., et al.: "Laparoscopic training on bench models.: "better and more cost effective than operating room experience?". J Am Coll Surg, 2000, 191, 272-283.

[19] Zheng B., Hur H.C., Johnson S., Swanström L.L.: "Validity of using fundamentals of laparoscopic surgery (FLS) program to assess laparoscopic competent for gynecologists". Surg Endosc, 2010, 24 , 152-160.

[20] Tang B., Tait I., Ross G., Chien P.: "Development and use of a restructured animal tissue model for training in laparoscopic salpingostomy and salpingectomy". J Minim Invasive Gynecol, 2011, 718, 85-791.

[21] Levine R.L., Kives S., Cathey G., Blinchevsky A., Acland R., Thompson C., et al.: "The use of lightly embalmed (fresh tissue) cadavers for resident laparoscopic training". J Minim Invasive Gynecol, 2006, 13, 451-456.

[22] Hoffman M.S., Ondrovic L.E., Wenham R.M., Apte S.M., Shames M.L., Zervos E.E., et al.: "Evaluation of the porcine model to teach various ancillary procedures to gynaecologic oncology fellows". $\mathrm{Am}$ J Obstet Gynecol, 2009, 201, 116.e1-3.

[23] Loukas C., Nikiteas N., Schizas D., Lahanas V., Georgiou E.: “A head-to-head comparison between virtual reality and physical reality simulation training for basic skills acquisition”. Surg Endosc, 2012, 26, 2550-2558.

[24] Iwata. N., Fujiwara. M., Kodera. Y., Tanaka. C., Ohashi. N., Nakayama. G., et al.: "Construct validity of the LapVR virtualreality surgical simulator". Surg Endosc, 2011, 25, 423-428.

[25] Loukas C., Nikiteas N., Kanakis M., Georgiou E.: "Deconstructing laparoscopic competence in a virtual reality simulation environment". Surgery, 2011, 149, 750-760.

[26] Watson D.I., Baigrie R.J., Jamieson G.G.: "A learning curve for laparoscopic fundoplication. Definable, avoidable, or a waste of time?". Ann Surg, 1996, 224, 198-203.
[27] MacFadyen B.V., Vecchio R., Ricardo A.E., Mathis C.R.: "Bile duct injury after laparoscopic cholecystectomy - The United States Experience". Surg Endosc, 1998, 12, 315-321.

[28] Eriksen J.R., Grantcharov T.: "Objective assessment of laparoscopic skills using a virtual reality stimulator". Surg Endosc, 2005 , 19, 1216-1219.

[29] Loukas C., Nikiteas N., Kanakis M., Georgiou E.: “The contribution of simulation training in enhancing key components of laparoscopic competence”. Am Surg, 2011, 77, 708-715.

[30] Hassan I., Zielke A.: "Is the aptitude of manual skills enough for assessing the training effect of students using a laparoscopic simulator?". Ger Med Sci, 2005, 3.

[31] Mansour S., Din N., Ratnasingham K., Irukulla S., Vasilikostas G. McCluney A.L., et al.: "FLS simulator performance predicts intraoperative laparoscopic skill”. Surg Endosc, 2007, 21, 1991-1995.

[32] Madan A.K., Harper J.L., Frantzides C.T., Tichansky D.S.: "Nonsurgical skills do not predict baseline scores in inanimate box or virtual-reality trainers". Surg Endosc, 2008, 22, 1686-1689.

[33] Grantcharov T.P., Bardram L., Funch-Jensen P., Rosenberg J.: "Impact of hand dominance, gender, and experience with computer games on performance in virtual reality laparoscopy". Surg Endosc, 2003, 17, 1082-1085.

[34] Lynch J., Aughwane P., Hammond T.M.: "Video games and surgical ability, a literature review". J Surg Educ, 2010, 67, 184-189.

[35] Varras M., Nikiteas N., Varra V.K., Varra F.N., Georgiou E., Loukas C.: "Role of laparoscopic simulators in the development and assessment of laparoscopic surgical skills in laparoscopic surgery and gynecology". World Acad Sci J., 2020, 2, 65-76.

[36] Papanikolaou I.G., Haidopoulos D., Paschopoulos M., Chatzipapas I., Loutradis D., Vlahos N.F.: "Changing the way we train surgeons in the 21th century: A narrative comparative review focused on box trainers and virtual reality simulators". Eur J Obstet Gynecol Reprod Biol, 2019, 235, 13-18.

[37] Torricelli F.C.M., Barbosa J.A., Marchini G.S.: "Impact of laparoscopic surgery training laboratory on surgeon's performance" World J Gastrointest Surg, 2016, 8, 735-743.

Corresponding Author:

MICHAIL VARRAS, M.D., M.Sc., Ph.D.

Fifth Department of Obstetrics and Gynecology,

'Elena Venizelou' General Maternity Hospital,

Athens (Greece)

e-mail: mnvarras@otenet.gr 